\title{
Nonoscillatory solutions to fourth-order neutral dynamic equations on time scales
}

\section{Yang-Cong Qiu ${ }^{1 *}$ (])}

\section{"Correspondence:}

q840410@qq.com

'School of Humanities, Shunde

Polytechnic, Foshan, P.R. China

\section{Springer}

\begin{abstract}
In this paper, we present some sufficient conditions and necessary conditions for the existence of nonoscillatory solutions to a class of fourth-order nonlinear neutral dynamic equations on time scales by employing Banach spaces and Krasnoselskii's fixed point theorem. Two examples are given to illustrate the applications of the results.
\end{abstract}

MSC: 34N05; 34C10; 39A13

Keywords: Nonoscillatory solution; Neutral dynamic equation; Fourth-order; Time scale

\section{Introduction}

In this paper, we consider the existence of nonoscillatory solutions to fourth-order nonlinear neutral dynamic equations of the form

$$
\left(r_{1}(t)\left(r_{2}(t)\left(r_{3}(t)(x(t)+p(t) x(g(t)))^{\Delta}\right)^{\Delta}\right)^{\Delta}\right)^{\Delta}+f(t, x(h(t)))=0
$$

on a time scale $\mathbb{T}$ satisfying sup $\mathbb{T}=\infty$, where $t \in\left[t_{0}, \infty\right)_{\mathbb{T}}$ with $t_{0} \in \mathbb{T}$.

The oscillation and nonoscillation of nonlinear differential and difference equations have been developed rapidly in the recent decades. Afterwards, the theory of time scale united the differential and difference ones, and since then many researchers have investigated the oscillation and nonoscillation criteria of nonlinear dynamic equations on time scales; see, for instance, the papers [1-19] and the references cited therein. The majority of the scholars obtained the sufficient conditions to ensure that the solutions of the equations oscillate or tend to zero by using the generalized Riccati transformation and integral averaging technique. The correlative research has made a great achievement. However, we note that there is not much research into the field of existence of nonoscillatory solutions to dynamic equations on time scales. Generally, a functional space and a fixed point theorem would be employed to analyze it. We refer the reader to [20-25] for details of the theory of time scale and to $[4-16,19]$ for the studies on the existence of nonoscillatory solutions to nonlinear neutral dynamic equations on time scales.

(c) The Author(s) 2019. This article is licensed under a Creative Commons Attribution 4.0 International License, which permits use sharing, adaptation, distribution and reproduction in any medium or format, as long as you give appropriate credit to the original author(s) and the source, provide a link to the Creative Commons licence, and indicate if changes were made. The images or other third party material in this article are included in the article's Creative Commons licence, unless indicated otherwise in a credit line to the material. If material is not included in the article's Creative Commons licence and your intended use is not permitted by statutory regulation or exceeds the permitted use, you will need to obtain permission directly from the copyright holder. To view a copy of this licence, visit http://creativecommons.org/licenses/by/4.0/. 
Definition 1.1 A solution $x$ to (1) is defined to be eventually positive (or eventually negative) if there exists $c \in \mathbb{T}$ such that $x(t)>0($ or $x(t)<0)$ on $[c, \infty)_{\mathbb{T}}$. If a solution is either eventually positive or eventually negative, then we say it is nonoscillatory.

For a class of $n$ th-order nonlinear neutral dynamic equations as follows:

$$
R_{n}(t, x(t))+f(t, x(h(t)))=0
$$

where

$$
R_{k}(t, x(t))= \begin{cases}z(t)=x(t)+p(t) x(g(t)), & k=0, \\ r_{n-k}(t) R_{k-1}^{\Delta}(t, x(t)), & 1 \leq k \leq n-1, \\ R_{n-1}^{\Delta}(t, x(t)), & k=n,\end{cases}
$$

some scholars had been concerned with the existence of nonoscillatory solutions to (2), successively. Without loss of generality, only the eventually positive solutions were discussed. Zhu and Wang [19] considered (2) for $n=1$. The authors introduced a Banach space

$$
\mathrm{BC}\left[T_{0}, \infty\right)_{\mathbb{T}}=\left\{x \in \mathrm{C}\left(\left[T_{0}, \infty\right)_{\mathbb{T}}, \mathbb{R}\right): \sup _{t \in\left[T_{0}, \infty\right)_{\mathbb{T}}}|x(t)|<\infty\right\}
$$

with the norm $\|x\|=\sup _{t \in\left[T_{0}, \infty\right)_{\mathbb{T}}}|x(t)|$, where $C\left(\left[T_{0}, \infty\right)_{\mathbb{T}}, \mathbb{R}\right)$ denotes all continuous functions mapping $\left[T_{0}, \infty\right)_{\mathbb{T}}$ into $\mathbb{R}$, and established the existence of nonoscillatory solutions to (2) by Krasnoselskii's fixed point theorem. Note that there exist only two cases for every eventually positive solution $x$ to (2): $\lim _{t \rightarrow \infty} x(t)=a>0$ or $\lim _{t \rightarrow \infty} x(t)=0$.

Gao and Wang [5] and Deng and Wang [4] investigated (2) for $n=2$ under different conditions $\int_{t_{0}}^{\infty} 1 / r_{1}(t) \Delta t<\infty$ and $\int_{t_{0}}^{\infty} 1 / r_{1}(t) \Delta t=\infty$, respectively. Gao and Wang [5] introduced the same Banach space as in [19] and concluded that all the eventually positive solutions to (2) converge to a positive constant or zero. Deng and Wang [4] defined an improved Banach space

$$
\mathrm{BC}_{\lambda}\left[T_{0}, \infty\right)_{\mathbb{T}}=\left\{x \in \mathrm{C}\left(\left[T_{0}, \infty\right)_{\mathbb{T}}, \mathbb{R}\right): \sup _{t \in\left[T_{0}, \infty\right)_{\mathbb{T}}}\left|\frac{x(t)}{R^{2 \lambda}(t)}\right|<\infty\right\}
$$

with the norm $\|x\|_{\lambda}=\sup _{t \in\left[T_{0}, \infty\right)_{\mathbb{T}}}\left|x(t) / R^{2 \lambda}(t)\right|$, where $\lambda=0,1$ and $R(t)=1+\int_{t_{0}}^{t} 1 / r_{1}(s) \Delta s$, and presented four cases for the eventually positive solution $x$ to (2): (i) $x \in A(0,0)$, (ii) $x \in A(b, 0)$ for some positive constant $b$, (iii) $x \in A(\infty, b)$ for some positive constant $b$, (iv) $\lim \sup _{t \rightarrow \infty} x(t)=\infty$ and $\lim _{t \rightarrow \infty} x(t) / R(t)=0$, where

$$
A(\alpha, \beta)=\left\{x: \lim _{t \rightarrow \infty} x(t)=\alpha \text { and } \lim _{t \rightarrow \infty} \frac{x(t)}{R(t)}=\beta\right\} .
$$

Therefore, it is not difficult to see that the existences of nonoscillatory solutions to (2) are greatly different for diverse cases of convergence and divergence of the integrals $\int_{t_{0}}^{\infty} 1 / r_{i}(t) \Delta t, i=1,2, \ldots, n-1$.

For $n=3$, Qiu [12] studied (2) under the assumption $\int_{t_{0}}^{\infty} 1 / r_{1}(t) \Delta t=\int_{t_{0}}^{\infty} 1 / r_{2}(t) \Delta t=\infty$ and summarized five cases for the eventually positive solutions to (2). To obtain the 
existence and asymptotic behavior of nonoscillatory solutions to (2) for other cases of $\int_{t_{0}}^{\infty} 1 / r_{i}(t) \Delta t, i=1,2$, Qiu et al. [16] were concerned with (2) under $\int_{t_{0}}^{\infty} 1 / r_{1}(t) \Delta t=\infty$ and $\int_{t_{0}}^{\infty} 1 / r_{2}(t) \Delta t<\infty$, while the case that $\int_{t_{0}}^{\infty} 1 / r_{1}(t) \Delta t<\infty$ and $\int_{t_{0}}^{\infty} 1 / r_{2}(t) \Delta t=\infty$ was considered in Qiu et al. [14]. There are four cases for the eventually positive solutions to (2). However, the existence and asymptotic behavior of the solutions are evidently different between [14] and [16].

Furthermore, for $n \geq 3$, Qiu and Wang [15] investigated (2) under the condition $\int_{t_{0}}^{\infty} 1 / r_{i}(t) \Delta t<\infty, i=1,2, \ldots, n-1$, and deduced that every eventually positive solution converges to a positive constant or zero, which complements and unites the results in [5, 19]. Qiu et al. [13] continued to study (2) with $\int_{t_{0}}^{\infty} 1 / r_{1}(t) \Delta t=\infty$ and $\int_{t_{0}}^{\infty} 1 / r_{i}(t) \Delta t<\infty$, $i=2,3, \ldots, n-1$. Four cases for the eventually positive solutions have also been presented, and the results are consistent with those in [16] when $n=3$.

In this paper, we consider the existence of nonoscillatory solutions to (1), which is (2) for $n=4$, under the following conditions:

(C1) $r_{1}, r_{2}, r_{3} \in \mathrm{C}_{\mathrm{rd}}\left(\left[t_{0}, \infty\right)_{\mathbb{T}},(0, \infty)\right)$ and there exist two positive constants $M_{1}$ and $M_{3}$ such that

$$
\int_{t_{0}}^{\infty} \frac{\Delta t}{r_{i}(t)}=M_{i}<\infty, \quad i=1,3 \quad \text { and } \quad \int_{t_{0}}^{\infty} \frac{\Delta t}{r_{2}(t)}=\infty
$$

(C2) $p \in \mathrm{C}_{\mathrm{rd}}\left(\left[t_{0}, \infty\right)_{\mathbb{T}}, \mathbb{R}\right)$ and $-1<\lim _{t \rightarrow \infty} p(t)=p_{0}<1$;

(C3) $g, h \in \mathrm{C}_{\mathrm{rd}}\left(\left[t_{0}, \infty\right)_{\mathbb{T}}, \mathbb{T}\right), g(t) \leq t$, and $\lim _{t \rightarrow \infty} g(t)=\lim _{t \rightarrow \infty} h(t)=\infty$; if $p_{0} \in(-1,0]$, then there exists a sequence $\left\{c_{k}\right\}_{k \geq 0}$ such that $\lim _{k \rightarrow \infty} c_{k}=\infty$ and $g\left(c_{k+1}\right)=c_{k}$;

(C4) $f \in \mathrm{C}\left(\left[t_{0}, \infty\right)_{\mathbb{T}} \times \mathbb{R}, \mathbb{R}\right), f(t, x)$ is nondecreasing in $x$, and $x f(t, x)>0$ for $x \neq 0$;

(C5) if

$$
\int_{t_{0}}^{\infty} \int_{t_{0}}^{u_{3}} \frac{\Delta u_{2} \Delta u_{3}}{r_{2}\left(u_{2}\right) r_{3}\left(u_{3}\right)}=\infty
$$

then we define

$$
R(t)=1+\int_{t_{0}}^{t} \int_{t_{0}}^{u_{3}} \frac{\Delta u_{2} \Delta u_{3}}{r_{2}\left(u_{2}\right) r_{3}\left(u_{3}\right)}
$$

where $\lim _{t \rightarrow \infty} R(g(t)) / R(t)=\eta \in(0,1]$ is satisfied.

In view of $(\mathrm{C} 1)$, it is clear that the results in the references are not available for (1). The conclusions in this paper can bring a deeper understanding of the existence and asymptotic behavior of nonoscillatory solutions to (2). In addition, we provide two significant examples to illustrate our results.

\section{Auxiliary results}

Firstly, we state Krasnoselskii's fixed point theorem (see [3]) as follows, which will be used in the sequel.

Lemma 2.1 Suppose that $X$ is a Banach space and $\Omega$ is a bounded, convex, and closed subset of X. If there exist two operators $U, V: \Omega \rightarrow X$ such that $U x+V y \in \Omega$ for all $x, y \in \Omega$, $U$ is a contraction mapping, and $V$ is completely continuous, then $U+V$ has a fixed point in $\Omega$. 
Then, we have another lemma to show the relationship between the functions $z$ and $x$. The proof is omitted since it is similar to those in [4, Lemma 2.3], [5, Theorem 1], [15, Lemma 2.3], and [19, Theorem 7].

Lemma 2.2 Suppose that $x$ is an eventually positive solution to (1) and

$$
\lim _{t \rightarrow \infty} \frac{z(t)}{R^{\lambda}(t)}=a, \quad \lambda=0,1
$$

where $\lambda=1$ only if (4) holds. If a is finite, then we have

$$
\lim _{t \rightarrow \infty} \frac{x(t)}{R^{\lambda}(t)}=\frac{a}{1+p_{0} \eta^{\lambda}}
$$

or $\lim \sup _{t \rightarrow \infty} x(t) / R^{\lambda}(t)=\infty$.

Finally, we need to divide all the eventually positive solutions to (1) into four groups for the sake of simplicity. In addition, define

$$
A(\alpha)=\left\{x \in S: \lim _{t \rightarrow \infty} x(t)=\infty \text { and } \lim _{t \rightarrow \infty} \frac{x(t)}{R(t)}=\alpha\right\}
$$

where $S$ is the set of all eventually positive solutions to (1).

Theorem 2.3 Suppose that $x$ is an eventually positive solution to (1). Then the solution $x$ belongs to one of the following four cases:

(A1) $\lim _{t \rightarrow \infty} x(t)=0$;

(A2) $\lim _{t \rightarrow \infty} x(t)=b$, where $b$ is a positive constant;

(A3) $x \in A(b)$, where $b$ is a positive constant;

(A4) $\lim \sup _{t \rightarrow \infty} x(t)=\infty$ and $\lim _{t \rightarrow \infty} x(t) / R(t)=0$.

Proof In view of (C2) and (C3), for any eventually positive solution $x$ to (1), there always exist $t_{1} \in\left[t_{0}, \infty\right)_{\mathbb{T}}$ and $p_{1}$ with $\left|p_{0}\right|<p_{1}<1$ such that $x(t)>0, x(g(t))>0, x(h(t))>0$, and $|p(t)| \leq p_{1}, t \in\left[t_{1}, \infty\right)_{\mathbb{T}}$. From (1) and $(C 4)$, for $t \in\left[t_{1}, \infty\right)_{\mathbb{T}}$, we have

$$
R_{4}(t, x(t))=R_{3}^{\Delta}(t, x(t))=-f(t, x(h(t)))<0,
$$

which means that $R_{3}$ is strictly decreasing on $\left[t_{1}, \infty\right)_{\mathbb{T}}$. Hence, it follows that

$$
R_{2}^{\Delta}(t, x(t)) \leq \frac{r_{1}\left(t_{1}\right) R_{2}^{\Delta}\left(t_{1}, x\left(t_{1}\right)\right)}{r_{1}(t)}, \quad t \in\left[t_{1}, \infty\right)_{\mathbb{T}} .
$$

From (5) we deduce that there are two cases for $R_{2}$. Assume that there exists $T \in\left[t_{1}, \infty\right)_{\mathbb{T}}$ satisfying $R_{2}^{\Delta}(T, x(T)) \leq 0$, then it is clear that $R_{2}^{\Delta}$ is eventually negative. If not, then we get $R_{2}^{\Delta}(t, x(t))>0, t \in\left[t_{1}, \infty\right)_{\mathbb{T}}$. That is, $R_{2}$ is always eventually monotonic. Substituting $s$ for $t$ in (5) and integrating (5) from $t_{1}$ to $t$, where $t \in\left[\sigma\left(t_{1}\right), \infty\right)_{\mathbb{T}}$, we obtain

$$
R_{2}(t, x(t))-R_{2}\left(t_{1}, x\left(t_{1}\right)\right) \leq r_{1}\left(t_{1}\right) R_{2}^{\Delta}\left(t_{1}, x\left(t_{1}\right)\right) \int_{t_{1}}^{t} \frac{\Delta s}{r_{1}(s)}
$$




$$
<r_{1}\left(t_{1}\right)\left|R_{2}^{\Delta}\left(t_{1}, x\left(t_{1}\right)\right)\right| \cdot M_{1}
$$

which implies that $R_{2}$ is upper bounded. Therefore, $R_{2}\left(\right.$ or $r_{2} R_{1}^{\Delta}$ ) is eventually monotonic and upper bounded. There are two cases to be discussed.

Case $1 . r_{2} R_{1}^{\Delta}$ is eventually decreasing. It follows that

$$
-\infty \leq \lim _{t \rightarrow \infty} r_{2}(t) R_{1}^{\Delta}(t, x(t))=L_{1}<\infty
$$

(a) If $-\infty \leq L_{1}<0$, then there exist $t_{2} \in\left[t_{1}, \infty\right)_{\mathbb{T}}$ and a constant $a_{1}<0$ such that $r_{2}(t) R_{1}^{\Delta}(t, x(t)) \leq a_{1}$ or

$$
R_{1}^{\Delta}(t, x(t)) \leq \frac{a_{1}}{r_{2}(t)}, \quad t \in\left[t_{2}, \infty\right)_{\mathbb{T}} .
$$

Letting $t$ be replaced by $s$ in (6) and integrating (6) from $t_{2}$ to $t$, where $t \in\left[\sigma\left(t_{2}\right), \infty\right)_{\mathbb{T}}$, we derive

$$
r_{3}(t) z^{\Delta}(t)=R_{1}(t, x(t)) \leq R_{1}\left(t_{2}, x\left(t_{2}\right)\right)+a_{1} \int_{t_{2}}^{t} \frac{\Delta s}{r_{2}(s)} \rightarrow-\infty, \quad t \rightarrow \infty,
$$

which means that $z^{\Delta}$ is eventually negative, and thus $z$ is eventually strictly decreasing. It is easy to see that $z$ is eventually positive or eventually negative, but we can claim that $z$ is eventually positive in terms of (C3). Assume not; then we have $-1<p_{0} \leq 0$. Moreover, there exists $t_{3} \in\left[t_{2}, \infty\right)_{\mathbb{T}}$ such that $x(t)<-p(t) x(g(t)) \leq p_{1} x(g(t))$ for $t \in\left[t_{3}, \infty\right)_{\mathbb{T}}$. Choose a positive integer $N$ satisfying $c_{k} \in\left[t_{3}, \infty\right)_{\mathbb{T}}$ for all $k \geq N$. For any $k>N$, we always have

$$
x\left(c_{k}\right)<p_{1} x\left(c_{k-1}\right)<p_{1}^{2} x\left(c_{k-2}\right)<\cdots<p_{1}^{k-N} x\left(c_{N}\right)
$$

which implies that $\lim _{k \rightarrow \infty} x\left(c_{k}\right)=\lim _{k \rightarrow \infty} z\left(c_{k}\right)=0$. It causes a contradiction, since $z$ is eventually strictly decreasing and eventually negative. Therefore, we deduce

$$
0 \leq \lim _{t \rightarrow \infty} z(t)=L_{0}<\infty
$$

By virtue of Lemma 2.2, it follows that case (A1) or (A2) holds.

(b) If $0<L_{1}<\infty$, then there exist $t_{4} \in\left[t_{1}, \infty\right)_{\mathbb{T}}$ and a constant $a_{2}>0$ such that $r_{2}(t) R_{1}^{\Delta}(t, x(t)) \geq a_{2}$ or

$$
R_{1}^{\Delta}(t, x(t)) \geq \frac{a_{2}}{r_{2}(t)}, \quad t \in\left[t_{4}, \infty\right)_{\mathbb{T}} .
$$

Similarly, we have

$$
r_{3}(t) z^{\Delta}(t)=R_{1}(t, x(t)) \geq R_{1}\left(t_{4}, x\left(t_{4}\right)\right)+a_{2} \int_{t_{4}}^{t} \frac{\Delta s}{r_{2}(s)} \rightarrow \infty, \quad t \rightarrow \infty .
$$

It means that $z^{\Delta}$ is eventually positive and thus $z$ is eventually strictly increasing. Similar to the proof in (a), we derive

$$
0 \leq \lim _{t \rightarrow \infty} z(t)=L_{0} \leq \infty
$$


If $L_{0}=\infty$, then we deduce

$$
\lim _{t \rightarrow \infty} r_{2}(t) R_{1}^{\Delta}(t, x(t))=\lim _{t \rightarrow \infty} \frac{z(t)}{R(t)}=L_{1}
$$

according to L'Hôpital's rule (see [24, Theorem 1.120]). By Lemma 2.2 we know that one of cases (A1)-(A3) holds.

(c) If $L_{1}=0$, since $r_{2} R_{1}^{\Delta}$ is eventually strictly decreasing, then $r_{2} R_{1}^{\Delta}$ and $R_{1}^{\Delta}$ are both eventually positive, and so $R_{1}$ (or $r_{3} z^{\Delta}$ ) is eventually strictly increasing. It follows that $r_{3} z^{\Delta}$ and $z^{\Delta}$ are both eventually positive or eventually negative, and $z$ is always eventually monotonic, which implies that $z$ is eventually positive or eventually negative. Similarly, we also obtain (7). In view of (C3), we see that one of cases (A1), (A2), and (A4) holds.

Case 2. $r_{2} R_{1}^{\Delta}$ is eventually increasing. It follows that

$$
-\infty<\lim _{t \rightarrow \infty} r_{2}(t) R_{1}^{\Delta}(t, x(t))=L_{1}<\infty .
$$

(a) If $-\infty<L_{1}<0$, then there also exist $t_{5} \in\left[t_{1}, \infty\right)_{\mathbb{T}}$ and a constant $a_{3}<0$ such that $r_{2}(t) R_{1}^{\Delta}(t, x(t)) \leq a_{3}$ for $t \in\left[t_{5}, \infty\right)_{\mathbb{T}}$. Similar to the proof in Case 1 , we get one of cases (A1) or (A2) holds.

(b) If $0<L_{1}<\infty$, similarly, then one of cases (A1)-(A3) holds.

(c) If $L_{1}=0$, since $r_{2} R_{1}^{\Delta}$ is eventually strictly increasing, then $r_{2} R_{1}^{\Delta}$ and $R_{1}^{\Delta}$ are both eventually negative. Hence, $R_{1}$ (or $r_{3} z^{\Delta}$ ) is eventually strictly decreasing. Similarly, we always have (7), and one of cases (A1), (A2), and (A4) holds.

The proof is complete.

\section{Sufficient conditions}

In this section, we firstly present some sufficient conditions for the existence of each type of eventually positive solutions to (1).

Theorem 3.1 If there exists some constant $K>0$ such that

$$
\int_{t_{0}}^{\infty} \int_{t_{0}}^{u_{3}} \int_{t_{0}}^{u_{2}} \int_{t_{0}}^{u_{1}} \frac{f\left(u_{0}, K\right)}{r_{1}\left(u_{1}\right) r_{2}\left(u_{2}\right) r_{3}\left(u_{3}\right)} \Delta u_{0} \Delta u_{1} \Delta u_{2} \Delta u_{3}<\infty
$$

then (1) has an eventually positive solution $x$ with $\lim _{t \rightarrow \infty} x(t)=b$, where $b$ is a positive constant.

Proof Suppose that there exists some constant $K>0$ satisfying (8). For $0 \leq p_{0}<1$, there are two cases $p_{0}>0$ and $p_{0}=0$. If $p_{0}>0$, then we choose a constant $p_{1}$ with $p_{0}<p_{1}<$ $\left(1+4 p_{0}\right) / 5<1$, and thus there exists $T_{0} \in\left[t_{0}, \infty\right)_{\mathbb{T}}$ such that for $t \in\left[T_{0}, \infty\right)_{\mathbb{T}}$, we have $p(t)>0,\left(5 p_{1}-1\right) / 4 \leq p(t) \leq p_{1}<1$, and

$$
\int_{T_{0}}^{\infty} \int_{T_{0}}^{u_{3}} \int_{T_{0}}^{u_{2}} \int_{T_{0}}^{u_{1}} \frac{f\left(u_{0}, K\right)}{r_{1}\left(u_{1}\right) r_{2}\left(u_{2}\right) r_{3}\left(u_{3}\right)} \Delta u_{0} \Delta u_{1} \Delta u_{2} \Delta u_{3} \leq \frac{\left(1-p_{1}\right) K}{8} .
$$

If $p_{0}=0$, then we take $p_{1}$ satisfying that $|p(t)| \leq p_{1} \leq 1 / 13$ for $t \in\left[T_{0}, \infty\right)_{\mathbb{T}}$.

Choose $T_{1} \in\left(T_{0}, \infty\right)_{\mathbb{T}}$ such that $g(t) \geq T_{0}$ and $h(t) \geq T_{0}$ for $t \in\left[T_{1}, \infty\right)_{\mathbb{T}}$. Let $\Omega_{1}=$ $\left\{x \in \mathrm{BC}_{0}\left[T_{0}, \infty\right)_{\mathbb{T}}: K / 2 \leq x(t) \leq K\right\}$, where $\mathrm{BC}_{0}\left[T_{0}, \infty\right)_{\mathbb{T}}$ is defined as (3) when $\lambda=0$, and 
$U_{1}, V_{1}: \Omega_{1} \rightarrow \mathrm{BC}_{0}\left[T_{0}, \infty\right)_{\mathbb{T}}$ as follows:

$$
\begin{aligned}
& \left(U_{1} x\right)(t)= \begin{cases}\left(U_{1} x\right)\left(T_{1}\right), & t \in\left[T_{0}, T_{1}\right)_{\mathbb{T}}, \\
3 K p_{1} / 4-p(t) x(g(t)), & t \in\left[T_{1}, \infty\right)_{\mathbb{T}},\end{cases} \\
& \left(V_{1} x\right)(t)= \begin{cases}\left(V_{1} x\right)\left(T_{1}\right), & t \in\left[T_{0}, T_{1}\right)_{\mathbb{T}}, \\
3 K / 4+\int_{t}^{\infty} \int_{T_{1}}^{u_{3}} \int_{T_{1}}^{u_{2}} \int_{T_{1}}^{u_{1}} \frac{f\left(u_{0}, x\left(h\left(u_{0}\right)\right)\right)}{r_{1}\left(u_{1}\right) r_{2}\left(u_{2}\right) r_{3}\left(u_{3}\right)} \Delta u_{0} \Delta u_{1} \Delta u_{2} \Delta u_{3}, & t \in\left[T_{1}, \infty\right)_{\mathbb{T}} .\end{cases}
\end{aligned}
$$

Similar to the proofs in [4, Theorem 2.5], [5, Theorem 2], [12, Theorem 3.1], [15, Theorem 3.1], and [19, Theorem 8], we omit the explanation that $U_{1}$ and $V_{1}$ satisfy the conditions in Lemma 2.1. Then there exists $x \in \Omega_{1}$ such that $\left(U_{1}+V_{1}\right) x=x$, which means that, for $t \in\left[T_{1}, \infty\right)_{\mathbb{T}}$, we obtain

$$
\begin{aligned}
x(t)= & \frac{3\left(1+p_{1}\right) K}{4}-p(t) x(g(t)) \\
& +\int_{t}^{\infty} \int_{T_{1}}^{u_{3}} \int_{T_{1}}^{u_{2}} \int_{T_{1}}^{u_{1}} \frac{f\left(u_{0}, x\left(h\left(u_{0}\right)\right)\right)}{r_{1}\left(u_{1}\right) r_{2}\left(u_{2}\right) r_{3}\left(u_{3}\right)} \Delta u_{0} \Delta u_{1} \Delta u_{2} \Delta u_{3} .
\end{aligned}
$$

Letting $t \rightarrow \infty$, from (C4) and Lemma 2.2, we deduce

$$
\lim _{t \rightarrow \infty} z(t)=\frac{3\left(1+p_{1}\right) K}{4} \text { and } \quad \lim _{t \rightarrow \infty} x(t)=\frac{3\left(1+p_{1}\right) K}{4\left(1+p_{0}\right)}>0 .
$$

For $-1<p_{0}<0$, change $p_{1}$ to satisfy $-p_{0}<p_{1}<\left(1-4 p_{0}\right) / 5<1$ and $\left(5 p_{1}-1\right) / 4 \leq-p(t) \leq$ $p_{1}<1$ for $t \in\left[T_{0}, \infty\right)_{\mathbb{T}}$. Let

$$
\left(\bar{U}_{1} x\right)(t)= \begin{cases}\left(\bar{U}_{1} x\right)\left(T_{1}\right), & t \in\left[T_{0}, T_{1}\right)_{\mathbb{T}}, \\ -3 K p_{1} / 4-p(t) x(g(t)), & t \in\left[T_{1}, \infty\right)_{\mathbb{T}} .\end{cases}
$$

Similarly, there also exists $x \in \Omega_{1}$ such that $\left(\bar{U}_{1}+V_{1}\right) x=x$, and we obtain

$$
\lim _{t \rightarrow \infty} z(t)=\frac{3\left(1-p_{1}\right) K}{4} \text { and } \lim _{t \rightarrow \infty} x(t)=\frac{3\left(1-p_{1}\right) K}{4\left(1+p_{0}\right)}>0 .
$$

This completes the proof.

Theorem 3.2 Assume that (4) holds. If there exists some constant $K>0$ such that

$$
\int_{t_{0}}^{\infty} \int_{t_{0}}^{u_{1}} \frac{f\left(u_{0}, K R\left(h\left(u_{0}\right)\right)\right)}{r_{1}\left(u_{1}\right)} \Delta u_{0} \Delta u_{1}<\infty
$$

then (1) has an eventually positive solution $x \in A(b)$, where $b$ is a positive constant.

Proof Suppose that there exists some constant $K>0$ such that (9) holds. Proceed as in the proof of Theorem 3.1, except that, for $p_{0}>0$, take $T_{0} \in\left[t_{0}, \infty\right)_{\mathbb{T}}$ satisfying $p(t)>0$, $\left(5 p_{1}-1\right) / 4 \leq p(t) \leq p_{1}<1, p(t) R(g(t)) / R(t) \geq\left(5 p_{1}-1\right) \eta / 4$ for $t \in\left[T_{0}, \infty\right)_{\mathbb{T}}$, and

$$
\int_{T_{0}}^{\infty} \int_{T_{0}}^{u_{1}} \frac{f\left(u_{0}, K R\left(h\left(u_{0}\right)\right)\right)}{r_{1}\left(u_{1}\right)} \Delta u_{0} \Delta u_{1} \leq \frac{\left(1-p_{1} \eta\right) K}{8} .
$$


Let $\Omega_{2}=\left\{x \in \mathrm{BC}_{1}\left[T_{0}, \infty\right)_{\mathbb{T}}: K R(t) / 2 \leq x(t) \leq K R(t)\right\}$, where $\mathrm{BC}_{1}\left[T_{0}, \infty\right)_{\mathbb{T}}$ is defined as (3) when $\lambda=1$, and $U_{2}, V_{2}: \Omega_{2} \rightarrow \mathrm{BC}_{1}\left[T_{0}, \infty\right)_{\mathbb{T}}$ as follows:

$$
\begin{aligned}
& \left(U_{2} x\right)(t)= \begin{cases}3 K p_{1} \eta R(t) / 4-p\left(T_{1}\right) x\left(g\left(T_{1}\right)\right) R(t) / R\left(T_{1}\right), & t \in\left[T_{0}, T_{1}\right)_{\mathbb{T}} \\
3 K p_{1} \eta R(t) / 4-p(t) x(g(t)), & t \in\left[T_{1}, \infty\right)_{\mathbb{T}}\end{cases} \\
& \left(V_{2} x\right)(t)=\left\{\begin{array}{lr}
3 K R(t) / 4, & t \in\left[T_{0}, T_{1}\right)_{\mathbb{T}}, \\
3 K R(t) / 4 & \\
\quad+\int_{T_{1}}^{t} \int_{T_{1}}^{u_{3}} \int_{u_{2}}^{\infty} \int_{T_{1}}^{u_{1}} \frac{f\left(u_{0}, x\left(h\left(u_{0}\right)\right)\right)}{r_{1}\left(u_{1}\right) r_{2}\left(u_{2}\right) r_{3}\left(u_{3}\right)} \Delta u_{0} \Delta u_{1} \Delta u_{2} \Delta u_{3}, & t \in\left[T_{1}, \infty\right)_{\mathbb{T}},
\end{array}\right.
\end{aligned}
$$

where $T_{1}$ is defined as in Theorem 3.1. Similarly, there exists $x \in \Omega_{2}$ such that $\left(U_{2}+V_{2}\right) x=$ $x$. For $t \in\left[T_{1}, \infty\right)_{\mathbb{T}}$, it follows that

$$
\begin{aligned}
x(t)= & \frac{3\left(1+p_{1} \eta\right) K R(t)}{4}-p(t) x(g(t)) \\
& +\int_{T_{1}}^{t} \int_{T_{1}}^{u_{3}} \int_{u_{2}}^{\infty} \int_{T_{1}}^{u_{1}} \frac{f\left(u_{0}, x\left(h\left(u_{0}\right)\right)\right)}{r_{1}\left(u_{1}\right) r_{2}\left(u_{2}\right) r_{3}\left(u_{3}\right)} \Delta u_{0} \Delta u_{1} \Delta u_{2} \Delta u_{3} .
\end{aligned}
$$

Letting $t \rightarrow \infty$, we derive

$$
\lim _{t \rightarrow \infty} \frac{z(t)}{R(t)}=\frac{3\left(1+p_{1} \eta\right) K}{4} \text { and } \quad \lim _{t \rightarrow \infty} \frac{x(t)}{R(t)}=\frac{3\left(1+p_{1} \eta\right) K}{4\left(1+p_{0} \eta\right)}>0
$$

For $-1<p_{0}<0$, similar to the proof in Theorem 3.1, we have

$$
\lim _{t \rightarrow \infty} \frac{z(t)}{R(t)}=\frac{3\left(1-p_{1} \eta\right) K}{4} \text { and } \lim _{t \rightarrow \infty} \frac{x(t)}{R(t)}=\frac{3\left(1-p_{1} \eta\right) K}{4\left(1+p_{0} \eta\right)}>0
$$

Moreover, it is clear that $\lim _{t \rightarrow \infty} x(t)=\infty$. The proof is complete.

Theorem 3.3 Assume that (4) holds. If there exists a positive constant $M$ satisfying that $|p(t) R(t)| \leq M$ for $t \in\left[t_{0}, \infty\right)_{\mathbb{T}}$,

$$
\int_{t_{0}}^{\infty} \int_{t_{0}}^{u_{1}} \frac{f\left(u_{0}, R\left(h\left(u_{0}\right)\right)\right)}{r_{1}\left(u_{1}\right)} \Delta u_{0} \Delta u_{1}<\infty
$$

and

$$
\int_{t_{0}}^{\infty} \int_{t_{0}}^{u_{3}} \int_{u_{2}}^{\infty} \int_{t_{0}}^{u_{1}} \frac{f\left(u_{0}, M+3 / 4\right)}{r_{1}\left(u_{1}\right) r_{2}\left(u_{2}\right) r_{3}\left(u_{3}\right)} \Delta u_{0} \Delta u_{1} \Delta u_{2} \Delta u_{3}=\infty
$$

then (1) has an eventually positive solution $x \in A(0)$.

Proof Suppose that there exists a constant $M>0$ such that $|p(t) R(t)| \leq M$ for $t \in\left[t_{0}, \infty\right)_{\mathbb{T}}$, and both of (10) and (11) hold. It is easy to see that $p_{0}=0$. There exist $T_{0} \in\left[t_{0}, \infty\right)_{\mathbb{T}}$ and $p_{1}$ with $0<p_{1}<1$ such that, for $t \in\left[T_{0}, \infty\right)_{\mathbb{T}}$, we have $|p(t)| \leq p_{1}<1,2 M+3 / 2 \leq R(t) / 4$, and

$$
\int_{T_{0}}^{\infty} \int_{T_{0}}^{u_{1}} \frac{f\left(u_{0}, R\left(h\left(u_{0}\right)\right)\right)}{r_{1}\left(u_{1}\right)} \Delta u_{0} \Delta u_{1} \leq \frac{1-p_{1}}{8}
$$


Let $\Omega_{3}=\left\{x \in \mathrm{BC}_{1}\left[T_{0}, \infty\right)_{\mathbb{T}}: M+3 / 4 \leq x(t) \leq R(t)\right\}$ and $U_{3}, V_{3}: \Omega_{3} \rightarrow \mathrm{BC}_{1}\left[T_{0}, \infty\right)_{\mathbb{T}}$ as follows:

$$
\begin{aligned}
& \left(U_{3} x\right)(t)= \begin{cases}M+3 / 4-p\left(T_{1}\right) x\left(g\left(T_{1}\right)\right) R(t) / R\left(T_{1}\right), & t \in\left[T_{0}, T_{1}\right)_{\mathbb{T}}, \\
M+3 / 4-p(t) x(g(t)), & t \in\left[T_{1}, \infty\right)_{\mathbb{T}},\end{cases} \\
& \left(V_{3} x\right)(t)= \begin{cases}M+3 / 4, & t \in\left[T_{0}, T_{1}\right)_{\mathbb{T}}, \\
M+3 / 4+\int_{T_{1}}^{t} \int_{T_{1}}^{u_{3}} \int_{u_{2}}^{\infty} \int_{T_{1}}^{u_{1}} \frac{f\left(u_{0}, x\left(h\left(u_{0}\right)\right)\right)}{r_{1}\left(u_{1}\right) r_{2}\left(u_{2}\right) r_{3}\left(u_{3}\right)} \Delta u_{0} \Delta u_{1} \Delta u_{2} \Delta u_{3}, & t \in\left[T_{1}, \infty\right)_{\mathbb{T}},\end{cases}
\end{aligned}
$$

where $T_{1}$ is defined as in Theorem 3.1. Similarly, there exists $x \in \Omega_{3}$ such that, for $t \in$ $\left[T_{1}, \infty\right)_{\mathbb{T}}$, we have

$$
\begin{aligned}
x(t)= & 2 M+\frac{3}{2}-p(t) x(g(t)) \\
& +\int_{T_{1}}^{t} \int_{T_{1}}^{u_{3}} \int_{u_{2}}^{\infty} \int_{T_{1}}^{u_{1}} \frac{f\left(u_{0}, x\left(h\left(u_{0}\right)\right)\right)}{r_{1}\left(u_{1}\right) r_{2}\left(u_{2}\right) r_{3}\left(u_{3}\right)} \Delta u_{0} \Delta u_{1} \Delta u_{2} \Delta u_{3} .
\end{aligned}
$$

Letting $t \rightarrow \infty$, it is not difficult to see that

$$
\lim _{t \rightarrow \infty} z(t)=\infty \quad \text { and } \quad \lim _{t \rightarrow \infty} \frac{z(t)}{R(t)}=0,
$$

which implies that

$$
\lim _{t \rightarrow \infty} x(t)=\infty \quad \text { and } \quad \lim _{t \rightarrow \infty} \frac{x(t)}{R(t)}=0
$$

since $|p(t) x(g(t))| \leq|p(t) R(t)| \leq M$ for $t \in\left[T_{1}, \infty\right)_{\mathbb{T}}$. This completes the proof.

Remark 3.4 It is not easy to find a sufficient condition for the existence of nonoscillatory solutions tending to zero to (1) since their asymptotic behaviors are more complex than those of other solutions. However, we refer the reader to [4, Theorem 2.8 and Remark 2.9], [5, Theorem 3], [12, Theorems 3.5 and 3.6], [15, Theorems 3.2 and 3.3], and [19, Theorems 9 and 10], where some instructive results are presented.

\section{Necessary conditions}

Some necessary conditions for the existence of eventually positive solutions to (1) are provided in this section, where an additional assumption is needed as follows:

$$
\int_{t_{0}}^{\infty} \int_{t_{0}}^{u_{3}} \int_{t_{0}}^{u_{2}} \frac{\Delta u_{1} \Delta u_{2} \Delta u_{3}}{r_{1}\left(u_{1}\right) r_{2}\left(u_{2}\right) r_{3}\left(u_{3}\right)}<\infty .
$$

Theorem 4.1 Assume that (12) holds and

$$
\int_{t_{0}}^{\infty} \int_{t_{0}}^{u_{3}} \frac{\Delta u_{2} \Delta u_{3}}{r_{2}\left(u_{2}\right) r_{3}\left(u_{3}\right)}<\infty .
$$

If (1) has an eventually positive solution $x$ with $\lim _{t \rightarrow \infty} x(t)=b$, where $b$ is a positive constant, then there exists some constant $K>0$ satisfying (8). 
Proof Suppose that (12) and (13) hold, and (1) has an eventually positive solution $x$ with $\lim _{t \rightarrow \infty} x(t)=b>0$. It follows that $\lim _{t \rightarrow \infty} z(t)=\left(1+p_{0}\right) b$, and there exists $t_{1} \in\left[t_{0}, \infty\right)_{\mathbb{T}}$ such that $x(t)>0, x(g(t))>0$, and $x(h(t)) \geq b / 2$ for $t \in\left[t_{1}, \infty\right)_{\mathbb{T}}$. Letting $t$ be replaced by $u_{0}$ in (1) and integrating (1) from $t_{1}$ to $u_{1}$, where $u_{1} \in\left[\sigma\left(t_{1}\right), \infty\right)_{\mathbb{T}}$, we have

$$
R_{3}\left(u_{1}, x\left(u_{1}\right)\right)=R_{3}\left(t_{1}, x\left(t_{1}\right)\right)-\int_{t_{1}}^{u_{1}} f\left(u_{0}, x\left(h\left(u_{0}\right)\right)\right) \Delta u_{0}
$$

and thus

$$
R_{2}^{\Delta}\left(u_{1}, x\left(u_{1}\right)\right)=\frac{R_{3}\left(t_{1}, x\left(t_{1}\right)\right)}{r_{1}\left(u_{1}\right)}-\int_{t_{1}}^{u_{1}} \frac{f\left(u_{0}, x\left(h\left(u_{0}\right)\right)\right)}{r_{1}\left(u_{1}\right)} \Delta u_{0} .
$$

Integrating (14) with respect to $u_{1}$ from $t_{1}$ to $u_{2}$, where $u_{2} \in\left[\sigma\left(t_{1}\right), \infty\right)_{\mathbb{T}}$, we have

$$
\begin{aligned}
R_{2}\left(u_{2}, x\left(u_{2}\right)\right)= & R_{2}\left(t_{1}, x\left(t_{1}\right)\right)+R_{3}\left(t_{1}, x\left(t_{1}\right)\right) \int_{t_{1}}^{u_{2}} \frac{\Delta u_{1}}{r_{1}\left(u_{1}\right)} \\
& -\int_{t_{1}}^{u_{2}} \int_{t_{1}}^{u_{1}} \frac{f\left(u_{0}, x\left(h\left(u_{0}\right)\right)\right)}{r_{1}\left(u_{1}\right)} \Delta u_{0} \Delta u_{1} .
\end{aligned}
$$

By analogy, we deduce

$$
\begin{aligned}
z(t)= & z\left(t_{1}\right)+R_{1}\left(t_{1}, x\left(t_{1}\right)\right) \int_{t_{1}}^{t} \frac{\Delta u_{3}}{r_{3}\left(u_{3}\right)}+R_{2}\left(t_{1}, x\left(t_{1}\right)\right) \int_{t_{1}}^{t} \int_{t_{1}}^{u_{3}} \frac{\Delta u_{2} \Delta u_{3}}{r_{2}\left(u_{2}\right) r_{3}\left(u_{3}\right)} \\
& +R_{3}\left(t_{1}, x\left(t_{1}\right)\right) \int_{t_{1}}^{t} \int_{t_{1}}^{u_{3}} \int_{t_{1}}^{u_{2}} \frac{\Delta u_{1} \Delta u_{2} \Delta u_{3}}{r_{1}\left(u_{1}\right) r_{2}\left(u_{2}\right) r_{3}\left(u_{3}\right)} \\
& -\int_{t_{1}}^{t} \int_{t_{1}}^{u_{3}} \int_{t_{1}}^{u_{2}} \int_{t_{1}}^{u_{1}} \frac{f\left(u_{0}, x\left(h\left(u_{0}\right)\right)\right)}{r_{1}\left(u_{1}\right) r_{2}\left(u_{2}\right) r_{3}\left(u_{3}\right)} \Delta u_{0} \Delta u_{1} \Delta u_{2} \Delta u_{3} .
\end{aligned}
$$

Letting $t \rightarrow \infty$, in terms of (C1), (C4), (12), (13), and the fact that $x(h(t)) \geq b / 2$ for $t \in$ $\left[t_{1}, \infty\right)_{\mathbb{T}}$, we conclude

$$
\int_{t_{1}}^{\infty} \int_{t_{1}}^{u_{3}} \int_{t_{1}}^{u_{2}} \int_{t_{1}}^{u_{1}} \frac{f\left(u_{0}, b / 2\right)}{r_{1}\left(u_{1}\right) r_{2}\left(u_{2}\right) r_{3}\left(u_{3}\right)} \Delta u_{0} \Delta u_{1} \Delta u_{2} \Delta u_{3}<\infty
$$

which means that (8) holds. The proof is complete.

Here is a lemma to present a sufficient condition to ensure that (4) is satisfied, and then Theorems 4.3 and 4.4 follow.

Lemma 4.2 Assume that (12) holds. If (1) has an eventually positive solution $x$ with $\lim _{t \rightarrow \infty} x(t)=\infty$, then (4) holds.

Proof If (1) has an eventually positive solution $x$ with $\lim _{t \rightarrow \infty} x(t)=\infty$, then we claim that $\lim _{t \rightarrow \infty} z(t)=\infty$. Assume not; by Theorem 2.3 we have $0 \leq \lim _{t \rightarrow \infty} z(t)<\infty$, which implies that $0 \leq \lim _{t \rightarrow \infty} x(t)<\infty$ in view of Lemma 2.2. Hence, we deduce $\lim _{t \rightarrow \infty} z(t)=$ $\infty$. Similar to the proof in Theorem 4.1, we arrive at (16). Letting $t \rightarrow \infty$, from (C1) and (12) we obtain (4) and complete the proof. 
Theorem 4.3 Assume that (12) holds. If (1) has an eventually positive solution $x \in A(b)$, where $b$ is a positive constant, then there exists some constant $K>0$ satisfying (9).

Proof Suppose that (12) holds and (1) has an eventually positive solution $x \in A(b)$, where $b$ is a positive constant, then (4) holds according to Lemma 4.2. Define $R$ as in (C5), by Lemma 2.2 and Theorem 2.3 we obtain

$$
\lim _{t \rightarrow \infty} z(t)=\infty \text { and } \quad \lim _{t \rightarrow \infty} R_{2}(t, x(t))=\lim _{t \rightarrow \infty} \frac{z(t)}{R(t)}=\left(1+p_{0} \eta\right) b
$$

Then there exists $t_{1} \in\left[t_{0}, \infty\right)_{\mathbb{T}}$ such that $x(t)>0, x(g(t))>0$, and $x(h(t)) \geq b R(h(t)) / 2$ for $t \in\left[t_{1}, \infty\right)_{\mathbb{T}}$. Proceeding as the proof in Theorem 4.1, we get (15). Letting $u_{2} \rightarrow \infty$, by $(C 1)$ and (C4) we conclude

$$
\int_{t_{1}}^{\infty} \int_{t_{1}}^{u_{1}} \frac{f\left(u_{0}, b R\left(h\left(u_{0}\right)\right) / 2\right)}{r_{1}\left(u_{1}\right)} \Delta u_{0} \Delta u_{1}<\infty
$$

which implies that (9) holds. The proof is complete.

Theorem 4.4 Assume that (12) holds. If (1) has an eventually positive solution $x \in A(0)$, then we have

$$
\int_{t_{0}}^{\infty} \int_{t_{0}}^{u_{1}} \frac{f\left(u_{0}, 3 / 4\right)}{r_{1}\left(u_{1}\right)} \Delta u_{0} \Delta u_{1}<\infty
$$

and

$$
\int_{t_{0}}^{\infty} \int_{t_{0}}^{u_{3}} \int_{u_{2}}^{\infty} \int_{t_{0}}^{u_{1}} \frac{f\left(u_{0}, R\left(h\left(u_{0}\right)\right)\right)}{r_{1}\left(u_{1}\right) r_{2}\left(u_{2}\right) r_{3}\left(u_{3}\right)} \Delta u_{0} \Delta u_{1} \Delta u_{2} \Delta u_{3}=\infty
$$

Proof Suppose that (12) holds and (1) has an eventually positive solution $x \in A(0)$. It follows that (4) holds, and we define $R$ as in (C5), then we obtain

$$
\lim _{t \rightarrow \infty} z(t)=\infty \quad \text { and } \quad \lim _{t \rightarrow \infty} R_{2}(t, x(t))=\lim _{t \rightarrow \infty} \frac{z(t)}{R(t)}=0 .
$$

There exists $t_{1} \in\left[t_{0}, \infty\right)_{\mathbb{T}}$ such that $3 / 4 \leq x(t) \leq R(t), 3 / 4 \leq x(g(t)) \leq R(g(t))$, and $3 / 4 \leq$ $x(h(t)) \leq R(h(t))$ for $t \in\left[t_{1}, \infty\right)_{\mathbb{T}}$. Similar to the proof in Theorem 4.1, we get (14). Integrating (14) with respect to $u_{1}$ from $t_{2}$ to $t$, where $t_{2} \in\left[\sigma\left(t_{1}\right), \infty\right)_{\mathbb{T}}$ and $t \in\left[\sigma\left(t_{2}\right), \infty\right)_{\mathbb{T}}$, we have

$$
\begin{aligned}
R_{2}(t, x(t))= & R_{2}\left(t_{2}, x\left(t_{2}\right)\right)+R_{3}\left(t_{1}, x\left(t_{1}\right)\right) \int_{t_{2}}^{t} \frac{\Delta u_{1}}{r_{1}\left(u_{1}\right)} \\
& -\int_{t_{2}}^{t} \int_{t_{1}}^{u_{1}} \frac{f\left(u_{0}, x\left(h\left(u_{0}\right)\right)\right)}{r_{1}\left(u_{1}\right)} \Delta u_{0} \Delta u_{1} .
\end{aligned}
$$

Letting $t \rightarrow \infty$, we obtain

$$
R_{2}\left(t_{2}, x\left(t_{2}\right)\right)=-R_{3}\left(t_{1}, x\left(t_{1}\right)\right) \int_{t_{2}}^{\infty} \frac{\Delta u_{1}}{r_{1}\left(u_{1}\right)}
$$




$$
+\int_{t_{2}}^{\infty} \int_{t_{1}}^{u_{1}} \frac{f\left(u_{0}, x\left(h\left(u_{0}\right)\right)\right)}{r_{1}\left(u_{1}\right)} \Delta u_{0} \Delta u_{1}
$$

According to (C1) and (C4), it follows that

$$
\int_{t_{2}}^{\infty} \int_{t_{1}}^{u_{1}} \frac{f\left(u_{0}, 3 / 4\right)}{r_{1}\left(u_{1}\right)} \Delta u_{0} \Delta u_{1}<\infty
$$

which implies that (17) holds. Substituting $u_{2}$ for $t_{2}$ in (19) and integrating (19) with respect to $u_{2}$ from $t_{1}$ to $u_{3}$, where $u_{3} \in\left[\sigma\left(t_{1}\right), \infty\right)_{\mathbb{T}}$, we have

$$
\begin{aligned}
R_{1}\left(u_{3}, x\left(u_{3}\right)\right)= & R_{1}\left(t_{1}, x\left(t_{1}\right)\right)-R_{3}\left(t_{1}, x\left(t_{1}\right)\right) \int_{t_{1}}^{u_{3}} \int_{u_{2}}^{\infty} \frac{\Delta u_{1} \Delta u_{2}}{r_{1}\left(u_{1}\right) r_{2}\left(u_{2}\right)} \\
& +\int_{t_{1}}^{u_{3}} \int_{u_{2}}^{\infty} \int_{t_{1}}^{u_{1}} \frac{f\left(u_{0}, x\left(h\left(u_{0}\right)\right)\right)}{r_{1}\left(u_{1}\right) r_{2}\left(u_{2}\right)} \Delta u_{0} \Delta u_{1} \Delta u_{2} .
\end{aligned}
$$

Similarly, it follows that

$$
\begin{aligned}
z(t)= & z\left(t_{1}\right)+R_{1}\left(t_{1}, x\left(t_{1}\right)\right) \int_{t_{1}}^{t} \frac{\Delta u_{3}}{r_{3}\left(u_{3}\right)} \\
& -R_{3}\left(t_{1}, x\left(t_{1}\right)\right) \int_{t_{1}}^{t} \int_{t_{1}}^{u_{3}} \int_{u_{2}}^{\infty} \frac{\Delta u_{1} \Delta u_{2} \Delta u_{3}}{r_{1}\left(u_{1}\right) r_{2}\left(u_{2}\right) r_{3}\left(u_{3}\right)} \\
& +\int_{t_{1}}^{t} \int_{t_{1}}^{u_{3}} \int_{u_{2}}^{\infty} \int_{t_{1}}^{u_{1}} \frac{f\left(u_{0}, x\left(h\left(u_{0}\right)\right)\right)}{r_{1}\left(u_{1}\right) r_{2}\left(u_{2}\right) r_{3}\left(u_{3}\right)} \Delta u_{0} \Delta u_{1} \Delta u_{2} \Delta u_{3} .
\end{aligned}
$$

Letting $t \rightarrow \infty$, we conclude that (18) holds and complete the proof.

Now, we can present some necessary and sufficient conditions for the existence of eventually positive solutions to (1). In fact, according to Theorems 3.1 and 4.1, we obtain the following corollary.

Corollary 4.5 Assume that (12) and (13) hold. Then (1) has an eventually positive solution $x$ with $\lim _{t \rightarrow \infty} x(t)=b>0$ if and only if there exists some constant $K>0$ satisfying (8).

Similarly, we get another corollary in terms of Theorems 3.2 and 4.3.

Corollary 4.6 Assume that (4) holds. Then (1) has an eventually positive solution $x \in A(b)$ if and only if there exists some constant $K>0$ satisfying (9), where $b$ is a positive constant.

\section{Examples}

To illustrate the applications of the conclusions in this paper, two interesting examples are presented as follows.

Example 5.1 Let $\mathbb{T}=\bigcup_{n=0}^{\infty}\left[3^{n}, 2 \cdot 3^{n}\right]$. For $t \in[3, \infty)_{\mathbb{T}}$, consider

$$
\left(t^{3}\left(\frac{1}{t^{\alpha}}\left(t^{\beta}\left(x(t)-\frac{t+\cos t}{3 t} x\left(\frac{t}{3}\right)\right)^{\Delta}\right)^{\Delta}\right)^{\Delta}\right)^{\Delta}+t^{2} x(3 t)=0 .
$$


Here, $r_{1}(t)=t^{3}, r_{2}(t)=1 / t^{\alpha}, r_{3}(t)=t^{\beta}, p(t)=-(t+\cos t) /(3 t), g(t)=t / 3, h(t)=3 t, f(t, x)=$ $t^{2} x, t_{0}=3$, and $p_{0}=-1 / 3$, where $\alpha \geq 0$ and $\beta>\alpha+3$. It is clear that conditions (C1) $-(C 4)$ are satisfied. Moreover, we have

$$
\begin{aligned}
& \int_{3}^{\infty} \int_{3}^{u_{3}} \int_{3}^{u_{2}} \int_{3}^{u_{1}} \frac{f\left(u_{0}, 1\right)}{r_{1}\left(u_{1}\right) r_{2}\left(u_{2}\right) r_{3}\left(u_{3}\right)} \Delta u_{0} \Delta u_{1} \Delta u_{2} \Delta u_{3} \\
& \quad=\int_{3}^{\infty} \int_{3}^{u_{3}} \int_{3}^{u_{2}} \int_{3}^{u_{1}} \frac{u_{0}^{2} u_{2}^{\alpha}}{u_{1}^{3} u_{3}^{\beta}} \Delta u_{0} \Delta u_{1} \Delta u_{2} \Delta u_{3} \\
& \quad<\frac{1}{3} \int_{3}^{\infty} \int_{3}^{u_{3}} \int_{3}^{u_{2}} \frac{u_{2}^{\alpha}}{u_{3}^{\beta}} \Delta u_{1} \Delta u_{2} \Delta u_{3}<\frac{1}{3} \int_{3}^{\infty} \int_{3}^{u_{3}} \frac{u_{2}^{\alpha+1}}{u_{3}^{\beta}} \Delta u_{2} \Delta u_{3} \\
& \quad<\frac{1}{3(\alpha+2)} \int_{3}^{\infty} \frac{\Delta u_{3}}{u_{3}^{\beta-\alpha-2}}<\infty .
\end{aligned}
$$

According to Theorem 3.1, we conclude that (20) has an eventually positive solution $x$ with $\lim _{t \rightarrow \infty} x(t)=b>0$.

Example 5.2 Let $\mathbb{T}=[1, \infty)_{\mathbb{R}}$. For $t \in[2, \infty)_{\mathbb{T}}$, consider

$$
\left(t^{\lambda}\left(\frac{1}{t}\left(t^{2}\left(x(t)+\frac{1}{2 t} x(t-1)\right)^{\prime}\right)^{\prime}\right)^{\prime}\right)^{\prime}+\frac{x(t)}{t^{2}}=0
$$

Here, we have $r_{1}(t)=t^{\lambda}, r_{2}(t)=1 / t, r_{3}(t)=t^{2}, p(t)=1 /(2 t), g(t)=t-1, h(t)=t, f(t, x)=x / t^{2}$, $t_{0}=2$, and $p_{0}=0$, where $1<\lambda \leq 2$. It is obvious that conditions (C1)-(C4) and (4) are satisfied. From (C5), we have

$$
R(t)=1+\int_{2}^{t} \int_{2}^{u_{3}} \frac{d u_{2} d u_{3}}{r_{2}\left(u_{2}\right) r_{3}\left(u_{3}\right)}=1+\int_{2}^{t} \int_{2}^{u_{3}} \frac{u_{2}}{u_{3}^{2}} d u_{2} d u_{3}=\frac{t}{2}-1+\frac{2}{t}
$$

which satisfies that $\eta=\lim _{t \rightarrow \infty} R(g(t)) / R(t)=1$. Moreover, we deduce

$$
\begin{aligned}
& |p(t) R(t)|=\frac{1}{2 t} \cdot\left(\frac{t}{2}-1+\frac{2}{t}\right) \leq M=\frac{1}{4}, \quad t \in[2, \infty)_{\mathbb{T}} \\
& \int_{2}^{\infty} \int_{2}^{u_{1}} \frac{f\left(u_{0}, R\left(h\left(u_{0}\right)\right)\right)}{r_{1}\left(u_{1}\right)} d u_{0} d u_{1} \\
& \quad \int_{2}^{\infty} \int_{2}^{u_{1}} \frac{u_{0} / 2-1+2 / u_{0}}{u_{0}^{2} u_{1}^{\lambda}} d u_{0} d u_{1} \\
& \quad<\frac{1}{2} \int_{2}^{\infty} \int_{2}^{u_{1}} \frac{1}{u_{0} u_{1}^{\lambda}} d u_{0} d u_{1}<\frac{1}{2} \int_{2}^{\infty} \frac{\ln u_{1}}{u_{1}^{\lambda}} d u_{1}<\infty
\end{aligned}
$$

and

$$
\begin{gathered}
\int_{2}^{\infty} \int_{2}^{u_{3}} \int_{u_{2}}^{\infty} \int_{2}^{u_{1}} \frac{f\left(u_{0}, M+3 / 4\right)}{r_{1}\left(u_{1}\right) r_{2}\left(u_{2}\right) r_{3}\left(u_{3}\right)} d u_{0} d u_{1} d u_{2} d u_{3} \\
\quad=\int_{2}^{\infty} \int_{2}^{u_{3}} \int_{u_{2}}^{\infty} \int_{2}^{u_{1}} \frac{u_{2}}{u_{0}^{2} u_{1}^{\lambda} u_{3}^{2}} d u_{0} d u_{1} d u_{2} d u_{3}=\infty
\end{gathered}
$$

By virtue of Theorems 3.2 and 3.3, we conclude that (21) has two eventually positive solutions $x_{1} \in A(b)$ for some positive constant $b$ and $x_{2} \in A(0)$. 
On the other hand, if we take $r_{3}(t)=t^{4}$ and $f(t, x)=t \cdot x$, but other functions remain unchanged in (21), then $(C 1)-(C 4)$ are still satisfied. In addition, we derive

$$
\int_{2}^{\infty} \int_{2}^{u_{3}} \int_{2}^{u_{2}} \frac{u_{2}}{u_{1}^{\lambda} u_{3}^{4}} d u_{1} d u_{2} d u_{3}<\infty \text { and } \int_{2}^{\infty} \int_{2}^{u_{3}} \frac{u_{2}}{u_{3}^{4}} d u_{2} d u_{3}<\infty
$$

which means that both of (12) and (13) hold. However, for all $K>0$, we always have

$$
\begin{gathered}
\int_{2}^{\infty} \int_{2}^{u_{3}} \int_{2}^{u_{2}} \int_{2}^{u_{1}} \frac{f\left(u_{0}, K\right)}{r_{1}\left(u_{1}\right) r_{2}\left(u_{2}\right) r_{3}\left(u_{3}\right)} d u_{0} d u_{1} d u_{2} d u_{3} \\
=K \int_{2}^{\infty} \int_{2}^{u_{3}} \int_{2}^{u_{2}} \int_{2}^{u_{1}} \frac{u_{0} u_{2}}{u_{1}^{\lambda} u_{3}^{4}} d u_{0} d u_{1} d u_{2} d u_{3}=\infty
\end{gathered}
$$

which implies that (8) is not satisfied. Therefore, we deduce that (21) has no eventually positive solution tending to a positive constant in terms of Theorem 4.1 or Corollary 4.5.

\section{Acknowledgements}

The author thanks the anonymous referees for their valuable suggestions.

\section{Funding}

This project was supported by the National Natural Science Foundation of China (11671406) and the Natural Science Program for Young Creative Talents of Innovation Enhancing College Project of Department of Education of Guangdong Province (2017GKQNCX111 and 2018-KJZX039).

\section{Availability of data and materials}

Not applicable.

\section{Competing interests}

The author declares that he has no competing interests.

\section{Authors' contributions}

All authors read and approved the final manuscript.

\section{Publisher's Note}

Springer Nature remains neutral with regard to jurisdictional claims in published maps and institutional affiliations.

Received: 30 October 2019 Accepted: 4 December 2019 Published online: 11 December 2019

\section{References}

1. Agarwal, R.P., Bohner, M., Li, T., Zhang, C.: Oscillation theorems for fourth-order half-linear delay dynamic equations with damping. Mediterr. J. Math. 11, 463-475 (2014)

2. Bohner, M., Hassan, T.S., Li, T.: Fite-Hille-Wintner-type oscillation criteria for second-order half-linear dynamic equations with deviating arguments. Indag. Math. 29, 548-560 (2018)

3. Chen, Y.: Existence of nonoscillatory solutions of $n$th order neutral delay differential equations. Funcialaj Ekvacioj 35 $557-570$ (1992)

4. Deng, X., Wang, Q.: Nonoscillatory solutions to second-order neutral functional dynamic equations on time scales. Commun. Appl. Anal. 18, 261-280 (2014)

5. Gao, J., Wang, Q.: Existence of nonoscillatory solutions to second-order nonlinear neutral dynamic equations on time scales. Rocky Mt. J. Math. 43, 1521-1535 (2013)

6. Karpuz, B.: Asymptotic behaviour of bounded solutions of a class of higher-order neutral dynamic equations. Appl. Math. Comput. 215, 2174-2183 (2009)

7. Karpuz, B.: Necessary and sufficient conditions on the asymptotic behaviour of second-order neutral delay dynamic equations with positive and negative coefficients. Math. Methods Appl. Sci. 37, 1219-1231 (2014)

8. Karpuz, B., Öcalan, Ö.: Necessary and sufficient conditions on asymptotic behaviour of solutions of forced neutral delay dynamic equations. Nonlinear Anal. 71, 3063-3071 (2009)

9. Karpuz, B., Öcalan, Ö., Rath, R.: Necessary and sufficient conditions for the oscillatory and asymptotic behaviour of solutions to neutral delay dynamic equations. Electron. J. Differ. Equ. 2009, 64 (2009)

10. Li, T., Han, Z., Sun, S., Yang, D.: Existence of nonoscillatory solutions to second-order neutral delay dynamic equations on time scales. Adv. Differ. Equ. 2009, 562329 (2009)

11. Li, T., Zhang, C., Thandapani, E.: Asymptotic behavior of fourth-order neutral dynamic equations with noncanonical operators. Taiwan. J. Math. 18, 1003-1019 (2014)

12. Qiu, Y.: Nonoscillatory solutions to third-order neutral dynamic equations on time scales. Adv. Differ. Equ. 2014, 309 (2014) 
13. Qiu, Y., Jadlovská, I., Lassoued, D., Li, T.: Nonoscillatory solutions to higher-order nonlinear neutral dynamic equations. Symmetry-Basel 11, 302 (2019)

14. Qiu, Y., Wang, H., Jiang, C., Li, T.: Existence of nonoscillatory solutions to third-order neutral functional dynamic equations on time scales. J. Nonlinear Sci. Appl. 11, 274-287 (2018)

15. Qiu, Y., Wang, Q.: Existence of nonoscillatory solutions to higher-order nonlinear neutral dynamic equations on time scales. Bull. Malays. Math. Sci. Soc. 41, 1935-1952 (2018)

16. Qiu, Y., Zada, A., Tang, S., Li, T.: Existence of nonoscillatory solutions to nonlinear third-order neutral dynamic equations on time scales. J. Nonlinear Sci. Appl. 10, 4352-4363 (2017)

17. Zhang, C., Agarwal, R.P., Bohner, M., Li, T.: Oscillation of fourth-order delay dynamic equations. Sci. China Math. 58, $143-160$ (2015)

18. Zhang, C., Li, T., Agarwal, R.P., Bohner, M.: Oscillation results for fourth-order nonlinear dynamic equations. Appl. Math Lett. 25, 2058-2065 (2012)

19. Zhu, Z., Wang, Q.: Existence of nonoscillatory solutions to neutral dynamic equations on time scales. J. Math. Anal. Appl. 335, 751-762 (2007)

20. Hilger, S.: Ein Maßkettenkalkül mit Anwendung auf Zentrumsmannigfaltigkeiten. Ph.D. thesis, Universität Würzburg (1988)

21. Hilger, S.: Analysis on measure chain—a unified approach to continuous and discrete calculus. Results Math. 18, 18-56 (1990)

22. Agarwal, R.P., Bohner, M.: Basic calculus on time scales and some of its applications. Results Math. 35, 3-22 (1999)

23. Agarwal, R.P., Bohner, M., O'Regan, D., Peterson, A.: Dynamic equations on time scales: a survey. J. Comput. Appl. Math. 141, 1-26 (2002)

24. Bohner, M., Peterson, A.: Dynamic Equations on Time Scales: An Introduction with Applications. Birkhäuser, Boston (2001)

25. Bohner, M., Peterson, A. (eds.): Advances in Dynamic Equations on Time Scales. Birkhäuser, Boston (2003)

\section{Submit your manuscript to a SpringerOpen ${ }^{\circ}$ journal and benefit from:}

- Convenient online submission

- Rigorous peer review

Open access: articles freely available online

- High visibility within the field

- Retaining the copyright to your article

Submit your next manuscript at $\boldsymbol{\nabla}$ springeropen.com 